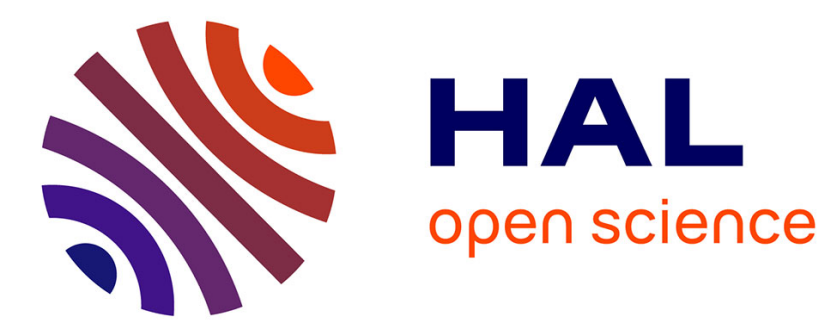

\title{
Looking for Efficient Implementations of Concurrent Objects
}

Achour Mostefaoui, Michel Raynal

\section{To cite this version:}

Achour Mostefaoui, Michel Raynal. Looking for Efficient Implementations of Concurrent Objects.

[Research Report] PI-1969, 2011, pp.10. inria-00576742

\section{HAL Id: inria-00576742 \\ https://hal.inria.fr/inria-00576742}

Submitted on 15 Mar 2011

HAL is a multi-disciplinary open access archive for the deposit and dissemination of scientific research documents, whether they are published or not. The documents may come from teaching and research institutions in France or abroad, or from public or private research centers.
L'archive ouverte pluridisciplinaire $\mathbf{H A L}$, est destinée au dépôt et à la diffusion de documents scientifiques de niveau recherche, publiés ou non, émanant des établissements d'enseignement et de recherche français ou étrangers, des laboratoires publics ou privés. 


\title{
Looking for Efficient Implementations of Concurrent Objects
}

\author{
Achour Mostefaoui ${ }^{*}$ Michel Raynal ${ }^{* *}$
}

\begin{abstract}
As introduced by Taubenfeld, a contention-sensitive implementation of a concurrent object is an implementation such that the overhead introduced by locking is eliminated in the common cases, i.e., when there is no contention or when the operations accessing concurrently the object are non-interfering. This paper, that can be considered as an introductory paper to this topic, presents a methodological construction of a contention-sensitive implementation of a concurrent stack. In a contention-free context a push or pop operation does not rest on a lock mechanism and needs only six accesses to the shared memory. In case of concurrency a single lock is required. Moreover, the implementation is starvation-free (any operation is eventually executed). The paper, that presents the algorithms in an incremental way, visits also a family of liveness conditions and important concurrency-related concepts such as the notion of an abortable object.
\end{abstract}

Key-words: Abortable object, Asynchronous shared memory system, Atomic register, Compare\&Swap, Contention manager, Contentionsensitiveness, Deadlock-freedom, Linearizability, Liveness, Lock-freedom, Non-blocking, Obstruction-freedom, Progress condition, Starvation-freedom, Synchronization.

\section{Sur la qualité de service des objects concurrents}

Résumé : Au delà de l'aspect recherche de solution de faisabilité, ce rapport surtout à la recherche de mise en ouvres efficaces d'objets concurrents.

Mots clés : Système asynchrone, mémoire partagée, objet concurrent, tolérance aux fautes.

\footnotetext{
${ }^{*}$ Membre du Projet ASAP: équipe commune avec l'INRIA, le CNRS et l'université Rennes 1, achour@irisa.fr.

*** Membre senior de l'IUF et Projet ASAP: équipe commune avec l'INRIA, le CNRS et l'université Rennes 1, raynal@irisa.fr.
} 


\section{Introduction}

\subsection{Concurrent objects}

From mastering sequential algorithms to mastering concurrency The study of algorithms lies at the core of informatics and participates in establishing it as a science with strong results on what can be computed (decidability) and what can be efficiently computed (complexity). It is consequently unanimously accepted by the community that any curriculum for undergraduate students has to include lectures on sequential algorithms. This allows the students not only to better master the basic concepts, mechanisms, techniques, difficulties and subtleties that underlie the design of algorithms, but also understand the deep nature of computer science and computer engineering.

A challenge is now to attain the same goal in the context of concurrency. A concurrent object is an object that can be concurrently accessed by several processes. As any object, a concurrent object is defined by a set of operations that processes can invoke to cooperate through this object. These operations are the only way to access the internal representation of the object (that remains otherwise invisible to processes). We are interested here in concurrent objects that have a sequential specification and supply processes with total operations. A total operation is an operation that always returns a result (e.g., instead of blocking the invoking process, a dequeue() operation on an empty queue returns it the value empty).

Linearizability The most popular safety property associated with concurrent objects is called linearizability [10]. This consistency condition extends atomicity to all objects defined by a sequential specification on total operations. More precisely, an implementation of an object satisfies linearizability (and we say that the object implementation is linearizable) if the operation invocations issued by the processes appear (from an external observer point of view) as if they have been executed sequentially, each invocation appearing as being executed instantaneously at some point of the time line between its start event and its end event. Said differently, an implementation is linearizable if it could have been produced by a sequential execution.

An important property associated with linearizable object implementations is that they compose for free. This means that, if both of the implementation of an object $A$ and the implementation of an object $B$ (each taken independently) are linearizable, then these implementations without any modification constitute a linearizable implementation of the composite object $(A, B)$. (It is important to notice that, in contrast to linearizability, other consistency conditions such as sequential consistency [14] or serializability [2] cannot be composed for free.)

Traditional lock-based shared memory synchronization One of the most popular way to obtain linearizable implementations of concurrent objects is to use locks. Associating a single lock with an object prevents several processes/threads from accessing it simultaneously. This approach is based on the classical notion of mutual exclusion $[3,18,24]$. Interestingly, locks can take different shapes according to the abstraction level at which they are considered. The most known example of locks is certainly the semaphore object [3], on top of which more friendly (i.e., high level) lock-based abstractions (such as monitors [12] or serializers [11]) can be built. This approach has proved its usefulness in providing simple lock-based solutions to basic paradigms of shared memory synchronization (such as the producer-consumer problem, or the readers-writers problem). One of the main difficulties when designing a lock-based solution lies in ensuring deadlock prevention, and more generally, provable liveness guarantees. Moreover, from an implementation point of view, lock implementations can be costly in terms of underlying shared memory accesses [19].

Contention-sensitive objects The notion of contention-sensitive implementation of a concurrent object has been recently introduced [26]. The contention-sensitiveness property means that the overhead due to locking has to be eliminated when there is no concurrency or when the operations that concurrently access an object are not interfering (e.g., enqueuing and dequeuing on a non-empty queue). In these cases (absence of contention or interference), a contention-sensitive implementation has to ensure that an operation on the object completes in a small (possibly constant) number of steps and without locks. Resorting to locks has to be restricted to concurrent conflicting operations only.

The first paper (to our knowledge) that introduced contention-sensitiveness (without giving it a name) is [16] where is presented a mutual exclusion algorithm in which, in a contention-free context, a process has to execute only seven shared memory accesses to enter the critical section. When there is contention, the number of shared memory accesses depends on the number of processes and the actual concurrency pattern.

\subsection{Content of the paper}

Abortable objects An abortable concurrent object behaves like an ordinary object when accessed sequentially, but may abort operations when accessed concurrently (in that case the aborted operation has no effect and returns a default value denoted $\perp$ ). This definition is inspired from, but stronger than, the definition of abortable objects introduced in [1] (in that paper, an aborted operation returns also $\perp$, but may or not take effect and this is not known by the invoking process). The important point (in both definitions) is that the state of the object is never left inconsistent. 
As far as we know, the notion of abortable objects has first been discussed in [13] where is presented an abortable mutual exclusion object. At any time while it is executing its entry code, a process can stop competing for the critical section and this halting has not to alter the liveness of the other critical section requests.

Progress conditions While it always considers linearizability as the implicit safety condition, this paper considers three progress conditions for concurrent objects: obstruction-freedom, non-blocking and starvation-freedom.

The obstruction-freedom progress condition [8] states that an operation is required to terminate only if it executes in a concurrencyfree context (i.e., when there is no operation invoked concurrently which is also called solo execution). Hence, an obstruction-free implementation of an object does not prevent concurrent operation invocations from never terminating. Let us notice that the notion of an abortable object is stronger than obstruction-freedom: while both ensure object consistency, they differ in the liveness they provide to users. More precisely, both guarantee operation termination in concurrency-free context, obstruction-freedom does not guarantee operation termination in case of concurrency. Differently, all operation invocations of an abortable object do terminate (possibly returning the value $\perp$ in case of concurrency). Hence, an implementation of an abortable object trivially satisfies the obstruction-freedom progress condition while the opposite is not true.

An implementation of a concurrent object is non-blocking if it is obstruction-free and additionally guarantees that, in presence of concurrency, at least one concurrent operation terminates. In a failure-free context, non-blocking is the same as deadlock-freedom. Finally, an implementation of a concurrent object is starvation-free if any operation invoked by a process terminates ${ }^{1}$. Hence, we have a hierarchy of progress conditions: obstruction-freedom is strictly weaker than non-blocking that in turn is strictly weaker than starvation-freedom. This hierarchy defines a family of qualities of service for liveness properties.

Content and roadmap This paper investigates the contention-sensitive approach for the implementation of concurrent objects as advocated by Taubenfeld in [26]. To that end, it considers a simple concurrent object, namely a shared task (let us remark that a lockbased starvation-free implementation of such an object is trivial). Three algorithms implementing such an object are presented. The first algorithm provides the processes with an abortable stack. As already said, this means that concurrent push and pop operations are allowed to abort (i.e., return $\perp$ ), while a push or pop operation executed in a concurrency-free context has to terminate and return a non- $\perp$ value. This algorithm does not use locks and is consequently lock-free. The second algorithm, which is also lock-free and provides the processes with a non-blocking shared stack is a simple extension of the previous one.

Considering an underlying abortable shared stack, the third algorithm provides the processes with a contention-sensitive shared stack. When an operation is executed in a concurrency-free context, this algorithm uses no lock and, whatever the number of processes and the size of the stack, it requires only seven shared memory accesses. This means that the algorithm is particularly efficient in contentionfree patterns. It resorts to a lock only when there are concurrent operations. Moreover, this algorithm ensures the starvation-freedom progress condition.

The algorithms are built incrementally. This helps better understand the mechanisms that are used to go from an abortable shared object to a contention-sensitive implementation that satisfies the starvation-freedom progress condition. Interestingly, the mechanism employed to ensure starvation-freedom constitute a contention manager that can be used to solve other fairness-related problems.

The paper is made up of 5 sections. Section 2 presents the computation model. Then Section 3 presents an algorithm implementing an abortable stack object and its extension to obtain a non-blocking implementation of a stack [22]. Section 4 presents a contentionsensitive algorithm that implements a starvation-free stack. This algorithm is based on a mechanism introduced in [26]. Finally, Section 5 concludes the paper. Last but not least, it is important to say that the aim of this paper is to promote the notion of contention-sensitive implementation of a concurrent object as an efficient alternative to fully lock-based implementations. The interested reader will find more general developments on the contention-sensitive approach in [26].

\section{Computation model}

\subsection{System model}

Asynchronous processes and communication model The system is made up of $n$ sequential processes denoted $p_{1}, p_{2}, \ldots, p_{n}$. The integer $i$ is the identity of $p_{i}$. Each process proceeds to its own speed, which means that the processes are asynchronous.

Processes communicate by accessing a shared memory that consists of atomic registers. The base operations on a register are read, write and Compare\&Swap (see below). "Atomic" means that all operations on a register $R$ appear as if they have been executed sequentially, and if operation $o p 1$ terminates before operation $o p 2$ starts, then $o p 1$ appears before $o p 2$ in the sequence.

Atomicity and linearizability denote the same consistency condition. The word "atomicity" is usually employed for read/write registers [15] while the word "linearizability" is employed for objects built on top of registers or other objects [10].

\footnotetext{
${ }^{1}$ In presence of process crashes, starvation-freedom becomes $t$-resilience where $t$ is the maximum number of process that may crash. Moreover, in a set of $n$ processes, wait-freedom [7] is $(n-1)$-resilience.
} 
Notation Shared registers are denoted with uppercase letters. In contrast, variables that are local to a process are denoted with lowercase letters.

Failure model It is assumed that both processes and atomic registers are reliable. This helps better understand how the algorithms work. They actually can cope with process crash failures. This is shortly discussed in Section 5.

\subsection{Compare\&Swap operation}

Definition The Compare\&Swap operation, that is on an atomic register $X$ is denoted $X$.C\&S (old, new). It is a conditional write that does atomically the following: if the current value of $X$ is old, it assigns new to $X$ and returns true; otherwise, it returns false.

primitive $X . C \& S($ old, new $)$ :

if $(X=$ old $)$ then $X \leftarrow$ new; return $($ true $)$ else return $($ false $)$ end if.

This base operation exists on some machines such as Motorola 680x0, Intel, Sun, IBM 370 and SPARC architectures. In some cases, the returned value is not a boolean, but the previous value of $X$.

The ABA problem When using Compare\&Swap, a process $p_{i}$ usually does the following. It first reads the atomic register $X$ (obtaining value $a$ ) and later wants to update $X$ to a new value $c$ only if $X$ has not been modified by another process since it has been read by $p_{i}$. Hence, $p_{i}$ invokes $X . C \& S(a, c)$. Unfortunately, the fact that this invocation returns true to $p_{i}$ does not allow it to conclude that $X$ has not been modified since the last time it read it. This is because between the read of $X$ and the invocation $X$.C\&S $(a, c)$ issued by $p_{i}, X$ may have been updated twice, first by a process $p_{j}$ that has successfully invoked $X$.C\&S $(a, b)$ and then by a process $p_{k}$ that has successfully invoked $X . C \& S(b, a)$, thereby restoring the value $a$ into $X$. This is called the ABA problem.

This problem can be solved by associating a new (tag) sequence number with each value that is written. The atomic register $X$ is then composed of several fields such as $\langle v, s n\rangle$ where $v$ is the current value of $X$ and $s n$ its associated sequence number. When it reads $X$ a process $p_{i}$ obtains consequently the pair $\langle v, s n\rangle$. When later it wants to conditionally writes $v^{\prime}$ into $X$, it invokes $X . \mathrm{C} \& \mathrm{~S}\left(\langle v, s n\rangle,\left\langle v^{\prime}, s n+1\right\rangle\right)$. It is easy to see that the write succeeds only if $X$ has continuously been equal to $\langle v, s n\rangle$.

\section{Implementing an abortable stack and a non-blocking stack}

The algorithm described in Figure 1 implements an abortable stack. It is a simplified version of the non-blocking algorithm introduced in [22] (which is presented in Figure 2).

Operations An abortable stack has two operations denoted here weak_push $(v)$ (where $v$ is the value to be added at the top of the stack) and weak_pop () . An operation always succeeds when executed in a contention-free context. In that case weak_push $(v)$ returns done if $v$ has been pushed on the stack and full if the stack is full; weak_pop() returns the value that was at the top of the stack (and suppresses it from the stack) or returns empty if the stack is empty. In the other cases, an operation may abort, in which case it returns $\perp$.

Shared data structures The stack is implemented with an atomic register denoted TOP and an array of $k+1$ atomic registers denoted $S T A C K[0 . . k]$.

- $T O P$ has three fields that contain an index (to address an entry of $S T A C K$ ), a value and a counter. It is initialized to $\langle 0, \perp, 0\rangle$.

- Each atomic register $S T A C K[x]$ has two fields: $S T A C K[x] . v a l$ that contains a value, and $S T A C K[x]$.sn that contains a sequence number (used to prevent the ABA problem as far as $S T A C K[x]$ is concerned).

The capacity of the stack is $k$ and for $1 \leq x \leq k$ the register $S T A C K[x]$ is initialized to $\langle\perp, 0\rangle$. STACK[0] is a dummy entry initialized to $\langle\perp,-1\rangle$ that always contains the default value $\perp$.

The array $S T A C K$ is used to store the content of the stack, and the register TOP is used to store the index and the value of the element at the top of the stack. The content of both TOP and STACK $[x]$ is modified with the help of the Compare\&Swap operation. This operation is used to prevent erroneous modifications of the stack internal presentation.

The implementation is lazy in the sense that a stack operation assigns its new value to TOP and leave the corresponding modification of $S T A C K$ to the next stack operation. Hence, while on the one hand a stack operation is lazy, on the other hand it has to help terminate the previous stack operation. 


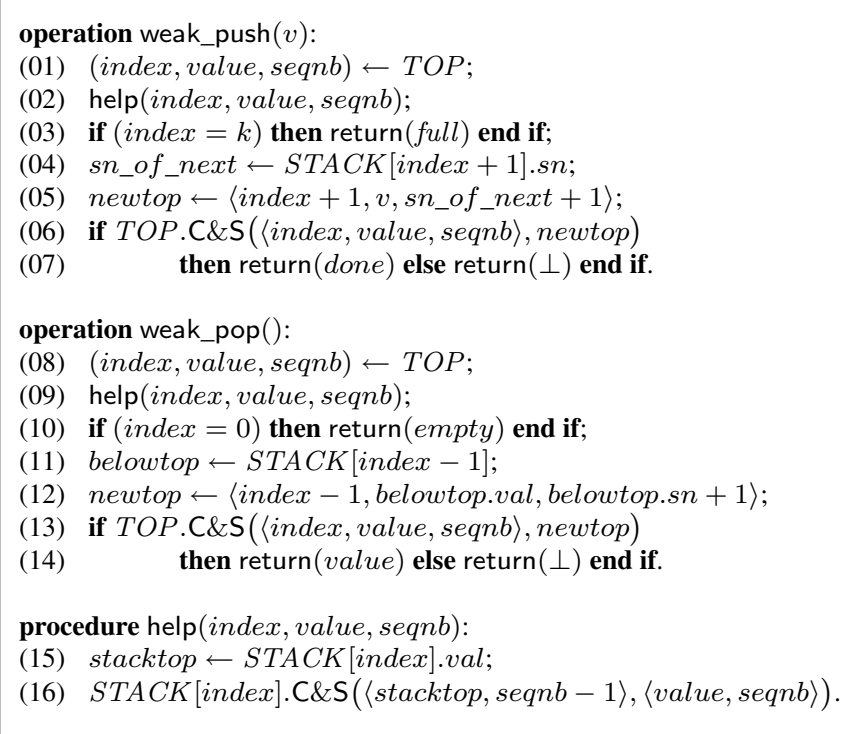

Figure 1: An abortable stack [22]

The operation weak_push $(v)$ When a process $p_{i}$ invokes weak_push $(v)$, it first reads the content of TOP (that contains the last non-aborted operation on the stack) and stores its three fields in its local variables index, value and seqnb (line 01).

Then, $p_{i}$ helps terminate the previous non-aborted stack operation (line 02). That operation (be it a successful weak_push() or a successful weak_pop () as we will see later) required to write $\langle$ value, seqnb $\rangle$ into $S T A C K[$ index $]$. To that end $p_{i}$ invokes $S T A C K[$ index $]$.C\&S. (old, new) with the appropriate values old and new in order the write be executed only if not yet done (lines $15-$ 16).

After its help (that was successful if not yet done by another stack operation) to move the content of TOP into STACK $\left[\right.$ index], $p_{i}$ returns full if the stack is full (line 03). If the stack is not full, it tries to modify TOP to register its push operation. This operation has to succeed if no other process modified TOP since it was read by $p_{i}$ at line 01 . In that case, TOP takes its new value and weak_push $(v)$ succeeds. Otherwise it aborts (lines 06-07).

The triple of values associated with this push_try $(v)$ and to be written in TOP if successful, is computed at lines (lines 04-05). Process $p_{i}$ first computes the last sequence number $s n_{-}$of_next used in $S T A C K[$ index +1$]$ and then defines the new triple, namely, newtop $=\langle$ index $+1, v$, sn_of_next +1$\rangle$ to be written first in TOP and later in $S T A C K[$ index +1$]$ thanks to the help provided by the next stack operation (let us remember that $s n_{-} o f \_n e x t+1$ is used to prevent the ABA problem).

The operation weak_pop () The algorithm implementing this operation has exactly the same structure as the previous one and is nearly the same. Its explanation is consequently left to the reader.

Linearization points of successful weak_push () and weak_pop () operations The operations that do not abort are linearizable, i.e., they can be totally ordered on the time line, each operation being associated with a single point of the time line that is after its start event and before its end event. More precisely, a non-aborted operation appears as if it has been atomically executed

- when it reads TOP (at line 01 or 08 ) if it returns full or empty (at line 03 or 10),

- or at the time at which it successfully executes TOP.C\&S $(-,-)$ (line 06 or 13 according to the operation).

From an abortable stack to a non-blocking stack A very simple algorithm that builds a non-blocking stack on top of an abortable stack is described in Figure 2. It is easy to see that this algorithm satisfies the obstruction-freedom property: an operation executed in a contention-free context returns always a non- $\perp$ value. It is also easy to see that no operation aborts: instead of aborting, an operation can loop forever. The interested reader will find in [22] a proof that, whatever the contention pattern, at least one operation always terminates (i.e., the algorithm is non-blocking). 


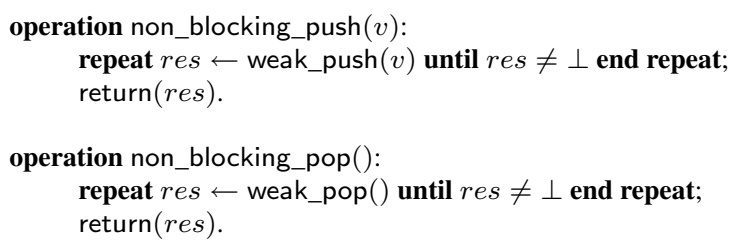

Figure 2: A linearizable non-blocking stack

\section{A contention-sensitive implementation of stack}

Let us remember that the aim is here the design of a contention-sensitive that implements a starvation-free stack, which means that the algorithm (a) is allowed to use a lock only when there is contention, and (b) has to execute a small and constant-bounded number of shared memory accesses when there is no contention.

The stack provides the processes with the operations denoted strong_push $(v)$ and strong_pop () . As the implementation of the contention-sensitiveness property is independent of the fact that the stack operation is strong_push() or strong_pop(), we describe a generic algorithm denoted strong_push_or_pop $($ par $)$ where $p a r=v$ if the operation is strong_push $(v)$ and $p a r=\perp$ if the operation is strong_pop () . Moreover, in the text of the algorithm weak_push_or_pop $($ par $)$ stands for weak_push $(v)$ or weak_pop () according to the context.

\subsection{Data structures}

The implementation of the contention-sensitiveness property is based on two atomic registers, an array of atomic registers and a lock.

- The lock, denoted $L O C K$, is accessed by the operations lock() and unlock(). It is used to ensure that a single process executes the part of code bracketed by $L O C K$.lock () and $L O C K$.unlock(). This lock is assumed to be deadlock-free but it is not required to be starvation-free (see the remark below).

- CONTENTION is a boolean register (initialized to false) that is set to true by a process when it executes the underlying weak_operation (par) operation. This allows a process that starts executing an operation to know that there is contention.

- FLAG $[i]$ boolean, is a boolean (initialized to false) that process $p_{i}$ sets to true when it wants to execute a stack operation and there is contention. In that way, $p_{i}$ allows the other processes to know it is competing for the lock. Process $p_{i}$ sets $F L A G[i]$ to false when it has executed its base weak_operation (par) operation.

- $T U R N$ contains a process identity. $T U R N=i$ means that process $p_{i}$ has priority to use the lock. Its initial value is any process identity. In order to ensure starvation freedom, the next value of $T U R N$ is $(T U R N \bmod n)+1$. Such a round-robin mechanism is used in several mutual exclusion algorithms such as [17, 23].

Remark If the lock is starvation-free (i.e., it ensures that any requesting process will obtain the lock) the algorithm can be simplified. More precisely, the array FLAG[1..n] and the register TURN become useless and consequently the lines 04-05 and 10-11 can be suppressed from algorithm. Those are actually shared variables and the associated statements that transform a deadlock-free lock into a starvation-free lock.

\subsection{The algorithm}

The algorithm is described in Figure 3. It is made of two parts. A lock-free part and a lock-based part. (The lock-free part is called shortcut in [26].)

In the first part (lines 01-03), the invoking process $p_{i}$ reads CONTENTION and, if this boolean is false, invokes the underlying weak_operation() operation. As we have seen if there is no contention this invocation returns a non- $\perp$ value and $p_{i}$ terminates. The number of shared memory accesses is then 6 (5 within the successful weak_push_or_pop ()$+1$ for the read of CONTENTION).

If CONTENTION is equal to true or weak_push_or_pop() returns $\perp, p_{i}$ knows there is contention. In that case, $p_{i}$ enters the second part (lines 04-13) which is made up of two phases.

- In the first phase (lines 04-05), $p_{i}$ first sets FLAG[i] to true to inform the other processes that it is competing for the critical section protected by the lock. Then, $p_{i}$ waits until either it is the process that is currently given priority $(T U R N=i)$ or the process that is currently given priority (namely $\left.p_{T U R N}\right)$ is not competing $(F L A G[T U R N]=$ false). When one of these predicates becomes true, $p_{i}$ invokes $L O C K$. lock(). 


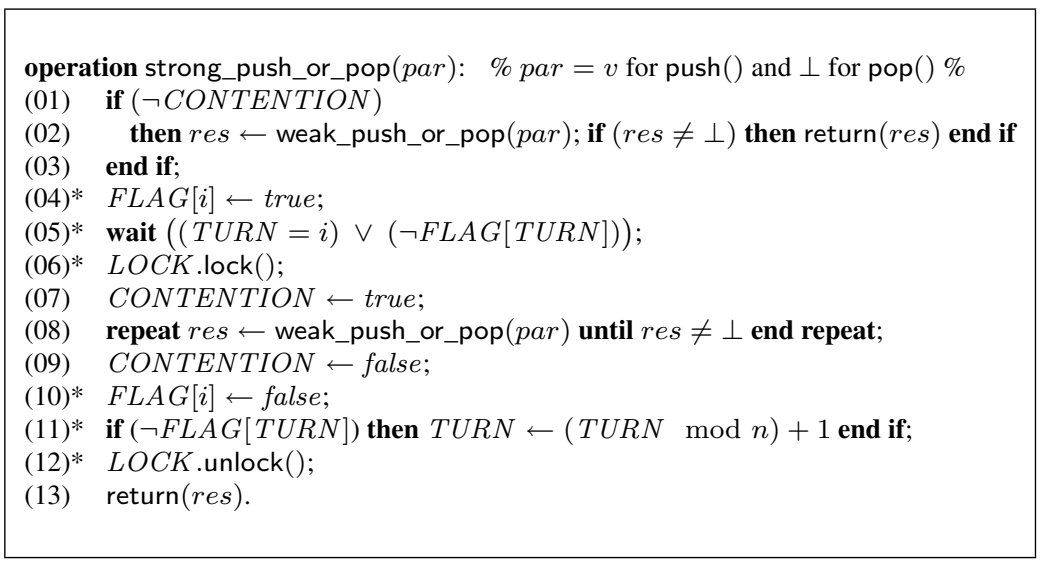

Figure 3: A linearizable contention-sensitive starvation-free stack (code for $p_{i}$ )

- The second phase (lines 06-13) starts when $p_{i}$ has gained mutual exclusion and is consequently the only process executing the lines 07-12.

Process $p_{i}$ executes then repeatedly weak_push_or_pop(par) until a successful invocation (line 08 ). When, this occurs it resets CONTENTION and FLAG[i] to false. Then if the process $p_{T U R N}$ that is currently given priority is not competing $\left(F L A G[T U R N]=\right.$ false), $p_{i}$ gives priority to $\left.p_{(T U R N} \bmod n\right)+1$ (line 11) before releasing the lock and returning its $($ non- $\perp)$ result.

It is important to notice that, due to asynchrony and the code of lines $01-03$, while a process $p_{i}$ is repeatedly executing weak_push_or_pop () at line 08 , other processes can be executing weak_push_or_pop() at line 02 (because they read false from CONTENTION) and the execution of weak_push_or_pop() by these processes can be successful. As we will see in the proof, this does not cause a problem because (a) the number of strong_push_or_pop() invocations concurrent with the one of $p_{i}$ is bounded and (b) the future invocations of strong_push_or_pop() will read true from CONTENTION) and will consequently enter the second part of the algorithm in which they cannot bypass $p_{i}$.

\subsection{Proof}

Lemma 1 If a process $p_{i}$ returns from its strong_push $(v)$ or strong_pop () invocation, it returns a non- $\perp$ value.

Proof The proof follows immediately from the predicate $r e s \neq \perp$ tested at line 02 if $p_{i}$ returns at that line, or tested at line 08 if $p_{i}$ returns line 13 .

Lemma 2 If a process $p_{i}$ eventually succeeds in locking, it eventually terminates its current strong_push() or strong_pop() operation.

Proof Let us assume that a process $p_{i}$ succeeds in locking at time $t_{1}$. There is a consequently a finite time $t_{2}>t_{1}$ from which CONTENTION is true.

It follows that all the processes that invoke strong_push $(v)$ or strong_pop () after time $t_{2}$ skip the lock-free part and start competing for the lock after it has been acquired by $p_{i}$. Hence these processes cannot prevent $p_{i}$ from terminating its operation.

It follows that at most $x$ processes, $0 \leq x \leq n-1$, can be executing weak_push() or weak_pop() at line 02 while $p_{i}$ is executing weak_push() or weak_pop() at line 08 . Let $X$ the corresponding set of processes. If $X=\emptyset$, the execution by $p_{i}$ of weak_push() or weak_pop () is concurrency-free and the the lemma trivially follows. Hence, let us consider the case $X \neq \emptyset$, As we have seen in Section 3 , the processes in $X$ eventually terminate their executions of weak_push() or weak_pop () . In the worst case, $p_{i}$ loops executing weak_push() or weak_pop() (at line 08) until all the processes in $X$ have returned from their current invocation of weak_push() or weak_pop () at line 02 . Let $t_{3}$ be such a time instant. If $p_{i}$ has not returned from its weak_push() or weak_pop() operation with a non- $\perp$ value before $t_{3}$, it follows from the previous observation that its first invocation of weak_push () or weak_pop () after $t_{3}$ will return a non- $\perp$ value, and consequently $p_{i}$ eventually terminates.

$\square$ Lemma 2

Lemma 3 If, while executing a strong_push $(v)$ or strong_pop() operation, a process $p_{i}$ reads true from CONTENTION at line 01 or obtains res $=\perp$ at line 02 , it eventually obtains the lock.

Proof Let us consider a process $p_{i}$ that sets $F L A G[i]$ to true (line 04 ). Hence, $p_{i}$ is a process as defined by the lemma assumption. We consider three cases. 
1. Process $p_{i}$ exits the loop of line 05 because $T U R N=i$.

Let us observe that, in this case, TURN remains equal to $i$ until $p_{i}$ resets $F L A G[i]$ to false (line 10) and increases TURN to $(i$ $\bmod n)+1($ line 11).

It follows from the previous observation that any process $p_{j}(j \neq i)$ that executes the loop of line 05 after TURN has been set to $i$, loops until $p_{i}$ executes the lines 10-11. Let $Y$ be this (possibly empty) set of processes.

Hence, at most $x$ processes, $0 \leq x \leq n-(|Y|+1)$, can compete with $p_{i}$ for obtaining the lock. As the lock is deadlock-free, it follows that, in the worst case, each of these processes obtains the lock before $p_{i}$. After they have obtained (and released) the lock, $p_{i}$ is the only process requesting the lock and necessarily obtains it, which completes the proof of the lemma for this case.

2. Process $p_{i}$ exits the loop of line 05 because $T U R N=k \neq i$ and $F L A G[k]$ is equal to false. We have to show that $p_{i}$ eventually obtains the lock.

Let us assume by contradiction that $p_{i}$ never obtains the lock. In the worst case, all processes are competing with $p_{i}$ to obtain the lock. Let $p_{j}$ be the process that obtains the lock (as the lock is deadlock-free, such a process does exist). Due to Lemma 2, it follows that $p_{j}$ eventually releases the lock. If FLAG[TURN] $=$ false, $p_{j}$ advances $T U R N$ to its successor $p_{\ell}$ (line 11 ) along the oriented logical ring $j, j+1, \ldots, n, 1, \ldots$ We have then $T U R N=\ell$. If $\ell \neq i$, the reasoning is repeated replacing $p_{j}$ by $p_{\ell}$ (let us observe that, due a reasoning similar to Item $1, p_{\ell}$ eventually obtains the look). As no process is skipped when $T U R N$ is advanced to its successor, it follows that TURN progresses from process to process until w have TURN $=i$. When this occurs, all processes that execute line 05 are blocked at that line until $p_{i}$ executes $F L A G[i] \leftarrow$ false (line 10).

It follows than, from then on, the number of processes competing with $p_{i}$ to obtain the lock is bounded. A reasoning similar to the used one in Item 1 shows that $p_{i}$ eventually obtains the lock, which contradicts the initial assumption and concludes the proof of the lemma for that case.

3. Process $p_{i}$ never exits the loop of line 05 . We show that this case cannot occur.

Let us assume by contradiction that $p_{i}$ loops forever at line 05 . This means that each time it evaluates the predicate at line 05 we have $T U R N \neq i \wedge F L A G[T U R N]$. Let $T U R N=k_{1}$ when read by $p_{i}$.

According to Item 1 and Item 2 , it follows that $p_{k_{1}}$ eventually exits the loop line at 05 because it finds $T U R N \neq k_{1}$ (Item 1 ) or $F L A G[T U R N]$ is false (Item 2) and consequently it eventually obtains the lock. Hence $p_{k_{1}}$ later executes $F L A G\left[k_{1}\right] \leftarrow$ false (line 10) and $T U R N \leftarrow\left(k_{1} \bmod n\right)+1$ (line 11). Let $k_{2}$ be that process identity. If $k_{2}=i$, $p_{i}$ exits the loop. Hence, let us

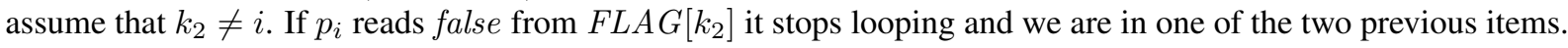

If $p_{i}$ reads always true from $F L A G\left[k_{2}\right]$, we are in the same case as previously, replacing $k_{1}$ by $k_{2}$. We consider then process $p_{k_{3}}$ such that $k_{3}=\left(k_{2} \bmod n\right)+1$. Etc.

If follows from the fact that no process is skipped when TURN is modified at line 10 that eventually $p_{i}$ either is such that $T U R N=i$ or reads false from $F L A G\left[k_{x}\right]$ for some process identity such that $T U R N=k_{x}$. When this happens we are in the case described in Item 1 or Item 2.

Theorem 1 Any invocation of strong_push() or strong_pop() operation returns a non- $\perp$ value, and all invocations are linearizable. Moreover, the algorithm is contention-sensitive: any strong_push() or strong_pop() operation invoked in a contention-free context is lock-free and accesses six times the shared memory.

Proof The fact any strong_push() or strong_pop() operation invoked in a contention-free context is lock-free and accesses six times the shared memory follows directly from the text of the algorithm.

The fact that no operation returns $\perp$ follows from Lemma 1 .

All invocations of strong_push() or strong_pop () that return at line 02 trivially terminate. The fact that all other invocations of strong_push() or strong_pop() terminate follows from Lemma 2 and Lemma 3.

The linearization point of a strong_push() (resp., strong_pop ()) operation is the linearization point of the last weak_push() (resp., weak_pop ()) operation it has executed (as defined in Section 3).

$\square$ Theorem 1

\subsection{From a non-blocking lock to a starvation-free lock}

When considering Figure 3, let us call starvation_free_lock $(i)$ the code defined by the starred lines 04-06 and starvation_free_unlock $(i)$ the code defined by the starred lines 10-12. The reader can notice that these two operations construct a starvation-free lock from a nonblocking one. The interested reader will find similar constructions in [23, 26]. 


\section{Concluding remarks}

Process crashes and unreliable registers When describing the previous algorithms which implement a concurrent task, we have considered that the processes where asynchronous but reliable. The reader can easily verify that these algorithms still work despite process crashes if no process crashes while holding the lock.

We have also assumed that the registers are reliable. Techniques to extend these algorithms to cope with unreliable registers have been studied in several works (e.g., [6]).

Contention managers Contention managers have recently become a hot research topic. The interested reader will find in $[4,25]$ techniques to extends obstruction-free or non-blocking algorithms to wait-free algorithms (wait-freedom is starvation-freedom in presence of any number of process crashes [7]). She will also find a failure detector-based approach to boost obstruction-freedom or non-blocking to wait-freedom in [5].

More generally, the interested reader will find developments on concurrent objects in [9, 20,21,24].

\section{References}

[1] Aguilera M.K., Frolund S., Hadzilacos V., Horn S.L. and Toueg S., Abortable and Query-abortable Objects and their Implementations. Proc. 26th Int'l ACM Symposium on Principles of Distributed Computing (PODC'07), pp. 23-32, 2007.

[2] Bernstein Ph.A., Hadzilacos V. and Goodman N., Concurrency Control and Recovery in Database Systems. Addison Wesley Publishing Company, 370 pages, 1987.

[3] Dijkstra E.W.D., Hierarchical Ordering of Sequential Processes. Acta Informatica, 1(1):115-138, 1971.

[4] Fich E.F., Luchangco V., Moir M. and Shavit N., Obstruction-free algorithms can be practically wait-free. Proc. 19th Int'l Symposium on Distributed Computing (DISC'05), Springer-Verlag LNCS \#3724, pp. 78-92, 2005.

[5] Guerraoui R., Kapalka M. and Kuznetsov P., The Weakest Failure Detectors to Boost Obstruction-freedom. Distributed Computing, 20(6): 415433, 2008.

[6] Guerraoui R. and Raynal M., From Unreliable Objects to Reliable Objects: the Case of Atomic Registers and Consensus. 9th Int'l Conference on Parallel Computing Technologies (PaCT'07), Springer Verlag LNCS LNCS \#4671, pp. 47-61, 2007.

[7] Herlihy M.P., Wait-Free Synchronization. ACM Transactions on Programming Languages and Systems, 13(1):124-149, 1991.

[8] Herlihy M.P., Luchangco V. and Moir M., Obstruction-free Synchronization: Double-ended Queues as an Example. Proc. 23th Int'l IEEE Conference on Distributed Computing Systems (ICDCS'03), pp. 522-529, 2003.

[9] Herlihy M.P. and Shavit N., The Art of Multiprocessor Programming, Morgan Kaufman Pub., San Francisco (CA), 508 pages, 2008.

[10] Herlihy M.P. and Wing J.M., Linearizability: a Correctness Condition for Concurrent Objects. ACM Transactions on Programming Languages and Systems, 12(3):463-492, 1990.

[11] Hewitt C.E. and Atkinson R.R., Specification and Proof Techniques for Serializers. IEEE Transactions on Software Engineering, SE5(1):1-21, 1979.

[12] Hoare C.A.R., Monitors: an Operating System Structuring Concept. Communications of the ACM, 17(10):549-557, 1974.

[13] Jayanti P., Adaptive and Efficient Abortable Mutual Exclusion. Proc. 22th Int'l ACM Symposium on Principles of Distributed Computing (PODC'03), pp. 295-304, 2003.

[14] Lamport L., How to Make a Multiprocessor Computer that Correctly Executes Multiprocess Programs. IEEE Transactions on Computers, C28(9):690-691, 1979.

[15] Lamport. L., On Interprocess Communication, Part 1: Basic formalism, Part II: Algorithms. Distributed Computing, 1(2):77-101,1986.

[16] Lamport L., A Fast Mutual Exclusion Algorithm. ACM Transactions on Computer Systems, 5(1):1-11, 1987.

[17] Peterson G.L., Myths about Mutual Exclusion. Information Processing Letters, 12(3):115-116, 1981.

[18] Raynal M., Algorithms for Mutual Exclusion. The MIT Press, ISBN 0-262-18119-3, 107 pages, 1986.

[19] Raynal M., Locks Considered Harmful: a Look at Non-traditional Synchronization. Proc. 6th Int'l Workshop on Software Technologies for Future Embedded and Ubiquitous Computing Systems (SEUS'08), Springer Verlag LNCS \#5287, pp. 369-380, 2008. 
[20] Raynal M., Shared Memory Synchronization in Presence of Failures: an Exercise-based Introduction. IEEE Int'l Conference on Complex, Intelligent and Software Intensive Systems (CISIS'09), IEEE Computer Society Press, pp. 9-18, Fukuoka (Japan), 2009.

[21] Raynal M., On the Implementation of Concurrent Objects. Tech Report \#1968, IRISA, Université de Rennes (France), 2011. To appear in a Springer Verlag LNCS special issue dedicated to the 75th birthday of Brian Randell.

[22] Shafiei N., Non-blocking Array-based Algorithms for Stacks and Queues. Proc. th Int'l Conference on Distributed Computing and Networking (ICDCN'09), Springer Verlag LNCS \#5408, pp. 55-66, 2009.

[23] Suzuki I. and Kasami T., A Distributed Mutual Exclusion Algorithm. ACM Transactions on Computer Systems, 3(4):344-349, 1985.

[24] Taubenfeld G., Synchronization Algorithms and Concurrent Programming. Pearson Prentice-Hall, ISBN 0-131-97259-6, 423 pages, 2006.

[25] Taubenfeld G., Efficient Transformations of Obstruction-free Algorithms into Non-blocking Algorithms. Proc. 21th Int'l Symposium on Distributed Computing (DISC'07), Springer-Verlag LNCS \#4731, pp. 450-464, 2007

[26] Taubenfeld G., Contention-Sensitive Data Structure and Algorithms. Proc. 23th Int'l Symposium on Distributed Computing (DISC'09), Springer Verlag LNCS \#5805, pp. 157-171, 2009. 